\title{
Inventário faunístico de um fragmento florestal urbano no Semiárido Brasileiro
}

\author{
Gleydson Vinicius dos Santos Silveira ${ }^{1 *}$, Zaíra dos Anjos Batista ${ }^{1}$, Carlos José da Silva $^{1}$, Natanael do \\ Nascimento Silva ${ }^{1}$, Hiago Renier Silva ${ }^{1}$
}

\begin{abstract}
RESUMO: O domínio da caatinga apresenta grande heterogeneidade de paisagem, o que contribui para abundância da fauna que, apesar de se encontrar em ambiente semiárido, apresenta grande diversidade de animais. Este estudo teve como objetivo inventariar as espécies da fauna ocorrentes no Parque municipal Maurício de Oliveira da cidade de Mossoró-RN. A área de estudo está dentro dos limites do parque ecológico urbano, localizada no centro da cidade, no Município de Mossoró no interior do estado do Rio Grande do Norte. Os dados do inventário rápido foram levantados no período de seis meses com coletas semanais. A busca visual deu-se percorrendo trilhas e áreas fora das trilhas do Parque, em horários diversificados. Foi registrado, o tipo de vestígio e quando avistados, o número de indivíduos. Para a constância de ocorrência das espécies foi adotado o índice de Constância (IC). Foi observado um total de 40 espécies de animais, sendo que, o número de espécies ocasionais apresentou um total de 16 espécies, já espécies consideradas acessórias tiveram um registro de 14 espécies e, as espécies constantemente apresentaram um total de 10 espécies. O reflorestamento com espécies frutíferas do bioma deve ser implantadas para que as espécies da fauna silvestre permanecer no ambiente.
\end{abstract}

Palavras-chave: Caatinga, Parque urbano, vertebrado

\section{Faunistic Inventory of an Urban Forest Fragment in the Brazilian Semi-Arid}

\begin{abstract}
The dominance of the caatinga presents great heterogeneity of landscape, which contributes to the abundance of the fauna that, despite being in a semi-arid environment, presents great diversity of animals. The objective of this study was to inventory the species of fauna occurring in the Maurício de Oliveira Municipal Park in the city of Mossoró (RN). The study area is within the limits of the urban ecological park, located in the center of the city, in the Municipality of Mossoró in the interior of the state of Rio Grande do Norte. Rapid inventory data were collected over the six-month period with weekly collections. The visual search was made through trails and areas outside the Park's tracks, at diversified times. The type of traces were recorded and, when sighted, the number of individuals. For the constancy of occurrence of the species, the Constância index (CI) was adopted. A total of 40 species of animals were observed, and the number of occasional species presented a total of 16 species, and species considered ancillary had a record of 14 species and, the species constantly presented a total of 10 species. Reforestation with biome fruit species should be deployed so that wildlife species remain in the environment.
\end{abstract}

Keywords: Caatinga, Urbanpark, Vertebrate

\section{INTRODUÇÃO}

A caatinga é constituída por fitofisionomias dotadas por um complexo vegetacional muito rico em espécies lenhosas caducifólias e herbáceas anuais que apresentam uma longa história de adaptação ao calor e a irregularidade do regime de chuvas, normalmente possuindo porte baixo, dossel descontínuo, folhas miúdas e indivíduos com muitas ramificações, geralmente com presença de espinhos ou acúleos para conter os efeitos de uma evapotranspiração muito intensa (AB'SABER, 1999; QUEIROZ, 2009).

O domínio da caatinga apresenta grande heterogeneidade de paisagem, o que contribui para abundância da fauna que, apesar de se encontrar em ambiente semiárido, apresenta grande diversidade de répteis, aves, mamíferos, anfíbios e invertebrados. Desta forma, ainda que a presença de animais em ambientes semiáridos seja menor do que em áreas mais úmidas, é necessário desmistificar que a caatinga seja um bioma com diversidade baixa em espécies e endemismo (PEREIRA, PEIXOTO, 2017).

O aumento da influência antrópica sobre as áreas naturais, afeta diretamente a conservação da biodiversidade desses locais e, com a expansão dos centros urbanos, tais ambientes ficam cada vez mais restritos a fragmentos isolados, sofrendo assim interferências em seus processos ecológicos e,

Recebido em 05/07/2019, Aceito para publicação em 15/10/2019

${ }^{1}$ EMBRAPA CENARGEN.

${ }^{2}$ Universidade Federal Rural do Semi-Árido (UFERSA)

*e-mail: gleydsonvinicius2013@gmail.com 
consequentemente, impactando de forma acelerada todos os fatores dependentes dela. Estudos em fragmentos florestais urbanos, geralmente com fatores de estresse inerentes, tais como a presença humana constante e a pouca diversificação de habitats, são necessários para que se possa catalogar as espécies remanescentes e assim planejar, de forma eficaz, ações de conservação para as mesmas. Este estudo teve como objetivo inventariar as espécies da fauna ocorrentes no Parque municipal Maurício de Oliveira da cidade de Mossoró-RN.

\section{MATERIAL E MÉTODOS}

A área de estudo está dentro dos limites do parque ecológico urbano Maurício de Oliveira, com uma área verde de 78 mil metros quadrados localizada no centro da cidade, às margens do Rio Mossoró, no Município de Mossoró no interior do estado do Rio Grande do Norte, na região Nordeste do Brasil.

Os dados do inventário rápido foram levantados no período de seis meses com coletas semanais. A busca visual deu-se percorrendo trilhas e áreas fora das trilhas do Parque, em horários diversificados, desde o horário do amanhecer até o anoitecer com duração de cerca de 2 horas cada turno. As áreas foram percorridas a pé em busca de registros diretos e indiretos como, visualizações, vestígios (rastros, fezes, pêlos, vocalizações, restos mortais) tomados pelos autores nas visitas semanais a área.

Foi registrado, o tipo de vestígio e quando avistados, o número de indivíduos. Todos os registros foram fotografados para posterior análise de identificação das espécies, consultando guias de campo. A nomenclatura adotada e a ordem de apresentação das espécies foram conforme (WILSON \&REEDER, 2005).

Para a constância de ocorrência das espécies foi adotado o índice de Constância (IC) de Silveira-Neto et al., (1976), utilizando a escala de Santos et al., (2008), Junior et al. (2013), na qual as espécies encontradas em mais de $50 \%$ das amostras são consideradas constantes; aquelas presentes entre 25 e $50 \%$ são acessórias e aquelas encontradas em menos de $25 \%$ das amostras são consideradas ocasionais.

\section{RESULTADOS E DISCUSSÃO}

O levantamento da fauna ocorrente no parque municipal de Mossoró apresentou uma distribuição taxonômica com elevado nível de classe com maior representatividade para o grupo das aves, seguida de répteis, mamíferos, peixes e por final, anfíbios

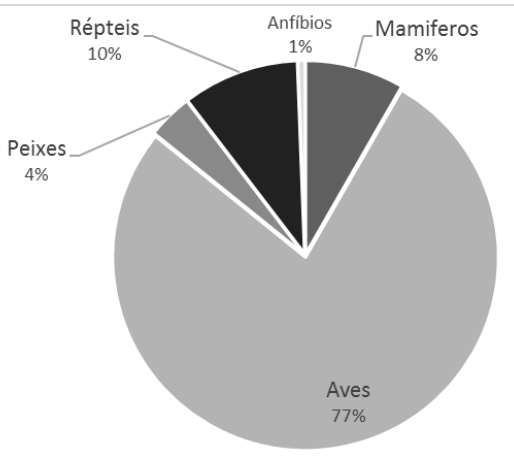

- Mamiferos | Aves | Peixes - Répteis " Anfibios

(Figura 1).

Figura 1-Distribuição das classes de animais registrados na pesquisa.

A tabela 1 apresenta alista de espécies bem como o modo de registro e o índice de constância. Pode-se observar durante a pesquisa um total de 40 espécies de animais, sendo que, o número de espécies ocasionais apresentou um total de 16 espécies, já espécies consideradas acessórias tiveram um registro de 14 espécies e, as espécies constantemente registradas apresentaram um total de 10 espécies (Figura 2).

Uma das justificativas para a maioria das espécies serem ocasionais deve-se a pouca variedade de espécies florísticas do bioma caatinga dentro do parque, o que possibilita poucos recursos alimentícios para os animais silvestres que forrageiam nesse ecossistema. Segundo Santos et al. (2017) a vegetação do parque é predominantemente exótica com predominância da espécie algaróba (Prosopisjuliflora (Sw.) DC.).

A fauna e a flora da caatinga passaram por diversos processos de modificações ao longo do tempo, o que resultou em imposição de diferentes comunidades de períodos distintos nesse espaço geográfico, pela observação das características fisionômicas da caatinga é possível observar a variedade de indivíduos que compõe a paisagem, as quais são resultantes do clima da região (LEAL et al., 2003).

Quanto à classificação taxonômica o morcego, a tartaruga de água doce e o lagarto não foram possíveis de serem identificadas quanto à espécie, uma vez que só a observação visual não foi suficiente para a identificação dos mesmos. A presença de uma tartaruga de água doce observada na área assim como espécies de peixes Tilápia podem ser em decorrência de solturas dessas espécies no rio Mossoró por moradores que habitam suas margens. As espécies Pitangussulphuratuse Crotophagaani foram observadas em todas as 
coletas, bem como aves aquáticas como Gallinulagaleatasendo assim classificadas como espécies constantes na área de estudo. Espécie aquática na área deve-se ao fato do parque está às margens do rio Mossoró.

Variações nos registros de coleta quanto aos horários de observação reforçam o período de atividade de cada grupo de animais, onde, no período observado pode-se constatar uma maior atividade de aves e mamíferos no início da manhã e final do dia, já os morcegos só foram observados ao findar do dia. Espécies como gaviões carcará usam a área para passarem a noite uma vez que espécies vegetais de alturas consideráveis como a Carnaúba e Algaroba servem de poleiro para esses animais se abrigarem no período noturno.

A presença de anfíbios do gênero Rhinella na caatinga é um dos indicadores que sustentam as hipóteses do processo de mudança que o bioma caatinga passou, já que essa espécie possui características fenotípicas de elasticidade que favorecem sua sobrevivência em períodos de estiagem e de altas temperaturas. (SIMON, 2010).

Tabela 1 - Listagem taxonômica, nome comum, forma de registro e índice de constância dos animais encontrados no Parque Maurício de Oliveira, Mossoró, Rio Grande do Norte. Índice de constância (IC): acessória (A), ocasional (O) e constante (C). Forma de registro: Visual (V), Canto (c).

\begin{tabular}{|c|c|c|c|}
\hline TAXON & NOME COMUM & FORMA DE REGISTRO & IC \\
\hline Troglodytesmusculus & Rouxinol & $\mathrm{V}$ & $\mathrm{C}$ \\
\hline Coerebaflaveola & Sibito & $\mathrm{Vec}$ & A \\
\hline Euphoniachlorotica & Vem-vem & $\mathrm{C}$ & A \\
\hline Guiraguira & Anu-branco & $\mathrm{V}$ & $\mathrm{O}$ \\
\hline Pitangussulphuratus & Bem-te-vi & $\mathrm{Cev}$ & $\mathrm{C}$ \\
\hline Eurolophosaurussp. & Lagarto & $\mathrm{V}$ & $\mathrm{C}$ \\
\hline Eupsittulacactorum & Periquito & $\mathrm{Vec}$ & A \\
\hline Crotophagaani & Anu-preto & $\mathrm{Vec}$ & $\mathrm{C}$ \\
\hline Ardeaalba & Garça-branca & V & $\mathrm{C}$ \\
\hline Xiphorhynchusatlanticus & Arapaçu rajado-do-nordeste & $\mathrm{V}$ & A \\
\hline Tachycinetaalbiventer & Andorinha & $\mathrm{V}$ & $\mathrm{O}$ \\
\hline Trochilidae & Beija-flor & V & A \\
\hline Harpagusbidentatus & Gavião & $\mathrm{C}$ & A \\
\hline Vanelluschilensis & Quero-quero & $\mathrm{Cev}$ & $\mathrm{O}$ \\
\hline Callithrixjacchus & Sagui-de-tufo-branco & V & $\mathrm{C}$ \\
\hline Testudines & Tartaruga de água doce & $\mathrm{V}$ & $\mathrm{O}$ \\
\hline Podilymbuspodiceps & Mergulhão & V & A \\
\hline Piculuschrysochloros & Pica-pau & V & $\mathrm{O}$ \\
\hline Megaceryletorquata & Pescador & V & A \\
\hline Butoridesstriatus & Socozinho & V & $\mathrm{C}$ \\
\hline Passermontanus & Pardal & V & A \\
\hline Tyrannus savana & Tesourinha & V & $\mathrm{O}$ \\
\hline Tilapiarendalli & Tilápia & V & A \\
\hline Columbina picui & Rolinha & V & A \\
\hline Columbina talpacoti & Rolinha roxa & V & $\mathrm{C}$ \\
\hline Felissilvestres catus & Gatodomético & V & $\mathrm{O}$ \\
\hline Gallinulagaleata & Galinha d'água & V & $\mathrm{C}$ \\
\hline Turdusrufiventris & Sabiá laranjeira & $\mathrm{C}$ & $\mathrm{O}$ \\
\hline Não identificado & Morcego & V & $\mathrm{C}$ \\
\hline Coragosatratus & Urubu & $\mathrm{V}$ & $\mathrm{O}$ \\
\hline Cerdocyonthous $l$. & Cachorro do mato & V & A \\
\hline Nettaerythrophthalma & Paturi- preto & V & A \\
\hline Paroaria dominicana & Cardeal-do-nordeste & $\mathrm{V}$ & $\mathrm{O}$ \\
\hline Mesoclemmystuberculata & Cágado & V & A \\
\hline Caracaraplancus & Caracará & V & $\mathrm{O}$ \\
\hline Athenecunicularia & Coruja & V & $\mathrm{O}$ \\
\hline Icterusjamacaii & Corrupião & V & $\mathrm{O}$ \\
\hline Rhinellaschneideri & Sapo cururu & $\mathrm{V}$ & $\mathrm{O}$ \\
\hline Dusicyonthous & Raposa & $\mathrm{V}$ & $\mathrm{O}$ \\
\hline
\end{tabular}

Fonte: Autores 


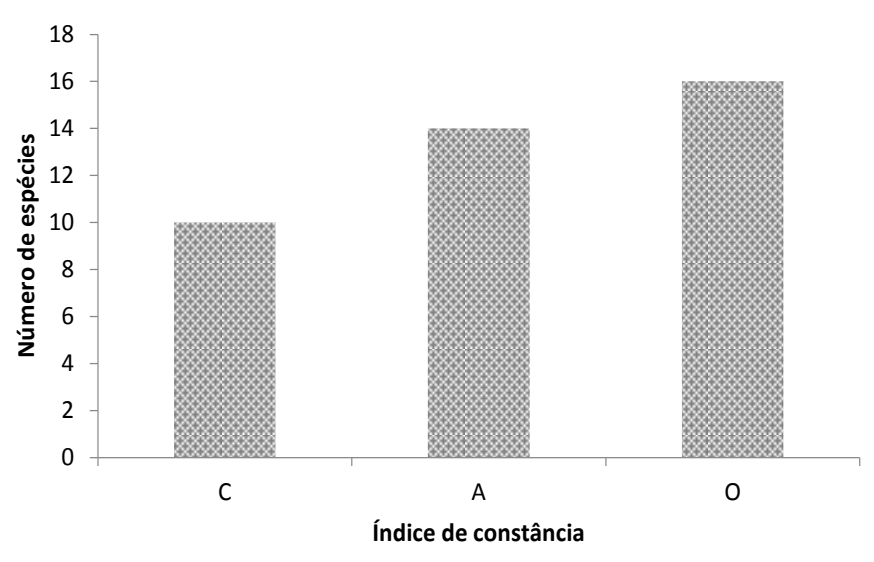

Figura 2 - Número de espécies de animais conforme o índice de constância: Acessória (A), Ocasional (O) e constante (C).

Espécies de mamíferos ameaçadas de extinção como a raposinha da caatinga (Dusicyonthous) foram registradas durante o período de observação. Essa espécie procura ovos de quelônios, pequenos mamíferos, repteis e ave para se alimentar. Animais domésticos como gatos também foram vistos em atividades de caça na área de estudo. A presença de animais domésticos se justifica uma vez que o parque está no perímetro urbano da cidade de Mossoró.

Lagartos do gênero Eurolophosaurus foram observados em todas as coletas apresentando muitos indivíduos visualizados durante o dia. Leal et al. (2003). Também observou hábito diurno para esse gênero. Caldas et al. (2015). Em concordância com Zug et al., (2001) e Vitt e Caldwell, (2014), fala que a densidade e a distribuição de uma população de lagartos refletem a disponibilidade de recursos no ambiente e é influenciada por eventos climáticos, efeitos de predação e presença ou ausência de habitats adequados. O mamífero que obteve um maior registro foi os saguis-de-tufos-brancos (Callithrixjacchus) Linnaeus, (1758) que pertencem à família Cebidae, subfamília Callitrichinae. Groves et al. (2005) e segundo Rylands (2012). Eles habitam florestas arbustivas da Caatinga e o Nordeste brasileiro. Tem aumentado bastante à quantidade de saguis em zonas urbanas, o que favorece o contato com a população humana. Eles podem habitar campos abandonados Raboyet al. (2008), sendo bem adaptado a habitats considerados marginais Kinzey (1997). Essa propriedade auxilia a espécie a invadir ambientes modificados antropicamente Ferrari (1996), e possibilita o estabelecimento de suas populações em parques, praças ou qualquer fragmento florestal, independente do seu tamanho, fato que também é favorecido pela conectividade natural entre espaços verdes urbanos (RUIZ-MIRANDA et al.,2006).

A presença de saguis em ambientes urbanos é uma realidade no Brasil. Brandão (2006) estima que aproximadamente 10.000 indivíduos coexistam com a população humana apenas no Rio de Janeiro. A atividade antrópica pode estar alterando a ecologia de várias espécies de calitriquídeos em geral Boere et al. (2000). Devido à grande sociabilidade dos calitriquídeos e ao seu domínio crescente dos espaços urbanos, a interação entre esses animais e humanos é cada vez mais comum.

De acordo com Dubauskas (2008), os saguis representam um problema potencial para as populações humanas e de outras espécies, competindo por alimentos e refúgios, gerando acidentes e sendo portadores de potenciais zoonoses e antropozoonoses. Por outro lado, existem interações positivas que podem ser ressaltadas, visto que os o encanto e simpatia dos saguis pode servir para atrair o interesse e o compromisso conservacionista tanto de moradores quanto de visitantes das regiões onde habitam. Todavia, as interações entre saguis e grupos humanos precisam ser esclarecidas, e esse contato deve ser contemplado nos planos e projetos das secretarias de Saúde, Educação e Turismo daqueles municípios, com informativos distribuídos para a população, para turistas em pousadas e outros locais de passagem dos grupos de saguis, e até mesmo ser incluso no cronograma de atividades da educação formal.

\section{CONCLUSÕES}

As aves foram o grupo de animais mais visualizados durante a pesquisa. Espécies de animais ameaçados de extinção como a raposinha da caatinga forrageiam no parque mesmo este estando dentro do perímetro urbano.

A Maioria das espécies observadas está classificada como ocasional isso indica que as mesmas buscam a área do parque para forragear em momentos específicos.

Animais domésticos como gatos partilham da mesma área de caça que os silvestres. Ação conservacionista como reflorestamento com espécies frutíferas do bioma deve ser implantada pelos gestores para que as espécies da fauna silvestre possam permanecer na área. 


\section{REFERÊNCIAS BIBLIOGRÁFICAS}

AB'SABER, A. N. Sertões e sertanejos: uma geografia humana sofrida. Revista Estudos Avançados, São Paulo, v. 13, n.36, p. 5-36, 1999.

BOERE, V.; TILLMANN, L.; RESENDE, M.C.; TOMAZ, C. Uso do Espaço e Comportamento Social em Saguis do Cerrado (Callithrixpenicillata) Selvagens no Centro de Primatologia da Universidade de Brasília. In: Alonso, C. e Lamgguth, A. A Primatologia no Brasil. Ed. Universitária /UFPB, p. 220. 2000.

BRANDÃO, C. A. Território e desenvolvimento: as múltiplas escalas entre o local e o global. Campinas,Editora da Unicamp, 2006.

CALDAS, F. L. S.; SANTANA, D. O.; SANTOS, R. A.; GOMES, F. F.A.; SILVA, B. D.; FARIA, R. G. Atividade e uso do espaço de Tropidurussemitaeniatus (Iguania) em área de Mata Atlântica, Nordeste do Brasil. Neotropical BiologyandConservation, 10(2): p. 85-92. 2015.

COIMBRA- FILHO, A.F.; ROCHA, N.D.C.; PISSINATTI, A. Morfofisiologia do ceco e sua correlação com o tipo odontológico em Callitrichidae (I Platyrrhini, Primates). RevistaBrasileira de Biologia. v.40, , p.177. 1980.

DUBAUSKAS, E. 2008. Fauna Brazilis. Home Page: www.faunabrazilis.com.br acessado em 16/09/2011.

Ecologia de Mabuyafrenata (Sauria, Scincidae) em uma área de afloramentos rochosos no sudeste do Brasil. Campinas, SP. Dissertação de mestrado. Universidade Estadual de Campinas, 72 p. 2000.

FERRARI, S.F. A vida secreta dos sagüis. Modelos do comportamento humano? Ciência Hoje, São Paulo, v. 20, p. 119.1996.

GROVES, C. In: REEDER, D.M.; WILSON, D.E. Mammal Species of the World. A Taxonomic and Geographic Reference. 3. ed. Johns Hopkins University Press, 2005.

JUNIOR, E. G.; GERALDO, C. H.; PERSEL, C.; FOGAÇA, P. L. C. Levantamento da mastofauna em fragmento florestal localizado na cidade de Cascavel Paraná. Cultivando o saber, Cascavel, v. 6, n. 4, p. 175 183. 2013.

KINZEY, W.G. Saguinus. In: KINZEY, W. G. New World Primates: Ecology, Evolution, and Behavior. New York, Aldine De Gruyter, p. 289. 1997.

LEAL, Inara Roberta; TABARELLI, Marcelo; DA SILVA, José Maria Cardoso. Ecologia e conservação da Caatinga. Editora Universitária UFPE, 2003.
Oliveira, Mossoró/RN. REVSBAU, Piracicaba, SP, v.12, n. 3, p. 94-106, 2017.

PEREIRA, C. J.; PEIXOTO, R. S. Levantamento de mamíferos terrestres em uma área de caatinga em Senhor do Bonfim, Bahia. Revista Brasileira de Zoociências 18(3): 33-44, 2017.

QUEIROZ, L. P. Leguminosas da caatinga. Feira de Santana: Universidade Estadual de Feira de Santana, 2009. 467 p.

RABOYET, B. E.; CANALE, G.R.; DIETZ J.M. Ecology of Callithrixkuhlii and a Review of Eastern Brazilian Marmosets.InternationalJournalofPrimatology, Washington, v. 29, p. 449-467. 2008.

Rylands, A. B., Brandon, K., 2005. Unidades de conservação brasileiras. Megadiversidade, 1(1), 27-35. 2012.

RUIZ-MIRANDA C. R.; AFONSO A.; MORAIS M.; VERONA C.; MARTINS A.; BECK B. Behavior and Ecological Interactions Between Introduced Golden-Lion Tamarins (Leontopithecusrosalia) and Introduced Marmosets (Callithrix sp.) in Brazil's Atlantic Coastal Forest Fragments. Brazilian Archives of Biology and Technology, Curitiba, v.49, n.1, p. 99-109.2006

SANTOS, P. C. S.; ALVES, L. L. B.; AQUINO, G. S. M.; ALMEIDA, G. N.; BOTREL, R. T.; CASTRO, V. G. Avaliação ambiental. P 88. 2008.

SILVEIRA-NETO, S., NAKANO, O. BARBIN, D.\& NOVA, N.A.V. Manual de ecologia dos insetos. EditoraAgronômica Ceres, São Paulo,1976, p.419.

SIMON, Monique Nouailhetas. Plasticidade fenotípica em relação à temperatura de larvas de Rhinella (Anura: Bufonidae) da caatinga e da floresta Atlântica.. Tese de Doutorado. Universidade de São Paulo. 2010.

VITT, L.J.; CALDWELL, J.P. 2014. Herpetology: an introductory biology of amphibians and reptiles, 4a ed., San Diego, Academic Press, 757 p. VRCIBRADIC, D. 1995.

WILSON, D.E. \& REEDER, D.M. Mammal species of the world: a taxonomic and geographic reference. 3 ed. The Johns Hopkins University Press, Baltimore, 2005, 2142p.

ZUG, G.R.; VITT, L.J.; CALDWELL, J.P..Herpetology: an introductory biology of. 2001; 2014. 